\title{
Biosensors and flow injection analysis
}

\section{Chien-Yuan Chen and Isao Karube}

\author{
National Taiwan University, Tapei, Taiwan and University of Tokyo, Tokyo, Japan
}

Combining flow injection analysis with a biosensor is a novel biosensing process which has allowed speedy and accurate analysis. Diagnostic analysis is the most important application for biosensing flow injection analysis, but other applications include bioprocess monitoring, analysis of food and agricultural products, as well as environmental analysis. In addition, the analysis of compounds, such as explosives and abused drugs, and monitoring of Salmonella, the microorganism that causes food poisoning, have been reported.

Current Opinion in Biotechnology 1992, 3:31-39

\section{Introduction}

It is difficult to give an exact definition of a biosensor. Widely speaking, a biosensor can be considered as an analytical device that responds to biological substances selectively and reversibly. According to this definition, any system that can be used to analyse a biological material can be classified as a biosensor. This definition, however, would include almost all physical and chemical sensors and is therefore obviously too wide. Thus, a more generally acceptable definition would be analytical devices that use a biological material as the sensing element.

Three elements are required for the construction of a biosensor (shown in Fig. 1): a recognition element composed of biological materials that react selectively with the specified substrate; a conversion element for converting the related information from the bio-catalytic reaction into electrical signals; and an element for recording these electrical signals.

The sensing elements used in biosensors can be generally classified into four groups: proteins, organelles, cells and tissues. Biosensors themselves can be divided into several groups according to the biological materials used or the reaction type involved, and a list is shown in Fig. 2. On the other hand, conversion elements used in biosensors employ most methods used in the fields of physical and chemical analysis. Spectrophotometry, amperometry, potentiometry, thermometry, fluorimetry and resonance luminescence are widely adopted for this purpose.

The use of biosensors started in 1962 when Clark and Lyons [1] combined an oxygen probe with glucose oxidase to determine glucose levels. The fact that biosensors still attract a lot of interest three decades after they were

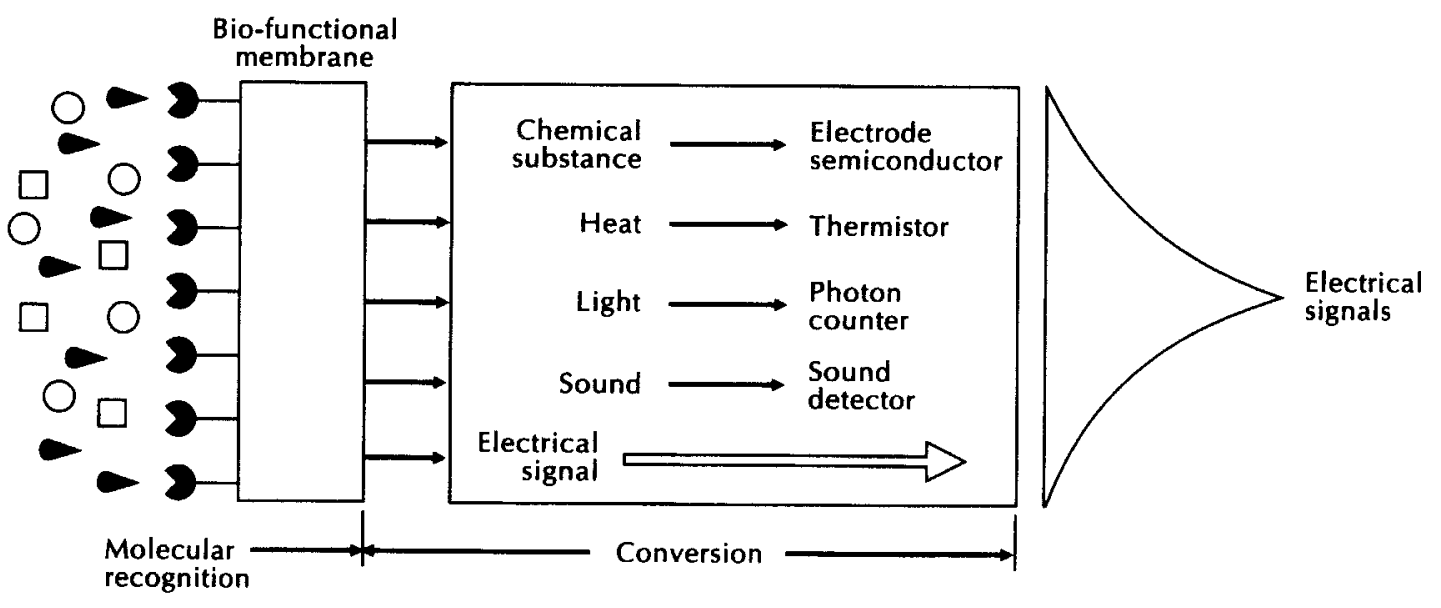

Fig.1. General biosensor construction and function. Chemical substances, heat,light, sound and electrical signals are produced during bio-catalyzed reactions in the bio-functional mwmbrane. These are converted into electrical signal which are then detected by electronic devices.

Abbreviations

FIA -flow injection analysis; G-6-P-glucose-6-phosphate; TNT-trinitrotoluene. 


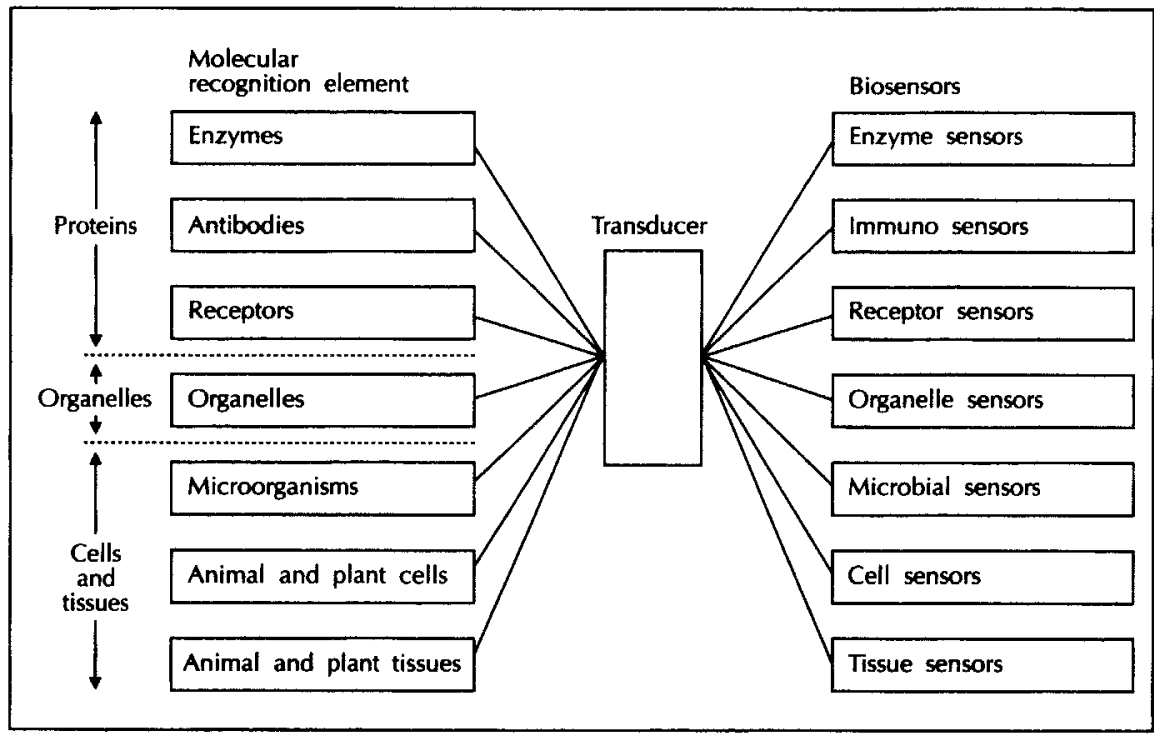

Fig. 2. Classification of biosensors according to the sensing elements used. Proteins, organelles, cells and tissues are major biomaterials used for constructing biosensors. first developed demonstrates their importance in many fields of analysis.

Although the technique of flow injection analysis (FIA) is not new, the combination of FIA with a detection device is novel in many analytical systems. There are still many studies concerned with the theory or operation of FIA. Some have tried to devise good methods for calibrating data and reducing interference [2]. Others have tried to establish mathematical models $[3,4]$, and still others have carried out error analysis of signals and then tried to establish a more reasonable operation process [5]. All of these have improved FIA to some extent. A biosensing FIA system is constructed by combining a biosensor with an FIA system. Most of the biological materials used as recognition elements in biosensors have the disadvantage of losing their activity when sterilized by heat or chemical treatment. However, a biosensor does not need to be sterilized if the sample is not returned to its source. Another problem is the decrease in response intensity if the biological material being used is kept in contact with its substrate for a long period of time. This can be overcome by using an FIA system to minimize the contact period between a biosensor and its substrate and to rinse the biological material with the carrying solution. In addition, it is possible to obtain almost-real-time data even though the sensor is not directly connected with the system under investigation, for example a fermenter. Other merits of a biosensing FIA system include the possibility of on-line calibration of the sensor and the manipulation of the sample before contact with the sensor.

\section{Glucose determination for clinical analysis}

Glucose determination is the most widely studied application of biosensors. This is partly because the high stability and satisfactory specificity of the glucose-related enzymes, including glucose oxidase and glucose dehydrogenase, make it quite easy to get good results. The major reason, however, is the applicability of glucose sensors to many fields, especially clinical analysis.

Glucose sensors have been studied for some decades and over this time have been much improved. Amperometric glucose enzyme sensors can be divided into three generations according to the electrodes and mediators used. The first generation sensors (Fig. 3) detect hydrogen peroxide formation or oxygen consumption in the reaction. The second generation sensors use a mediator to trans. fer electrons between the enzyme and the electrode and the third have an electrode made from a special material which allows direct transfer of electrons to it, from the enzyme. Conductive organic complex salts, for example Fulvadene-tetracyanoquinodimethane, have been used as electrode materials for this purpose. A recent study compared these three generations of sensor combined with FIA in determining glucose concentration in undiluted whole blood $\left[6^{\circ} \cdot\right.$. The second generation sensors seem to be better than the others, although the problem of mediator leakage from the electrode must be taken into consideration because most of the mediators used are small molecules that are difficult to immobilize together with enzyme.

Glucose sensors might also be improved by altering the materials and methods used for enzyme immobilization. Nylon has been used as the insoluble carrier for glucose oxidase immobilization and the effects of various spacers have been investigated in order to improve the efficiency of enzyme immobilization [7]. A nylon membrane with enzymes immobilized using spacers was combined with an FIA system and used to determine the concentration of glucose in blood serum [8]. Immobilization of enzymes in electropolymerized polypyrrole film on the surface of a platinum electrode was reported to provide a convenient sensor for flow injection glucose analysis [9॰]. The technique of electropolymerization has also been applied to prevent interference and electrode fouling [10]. If, however, whole blood instead of serum is used as the sample, the influence of the blood cells should be taken into account. This problem can be solved by improv- 


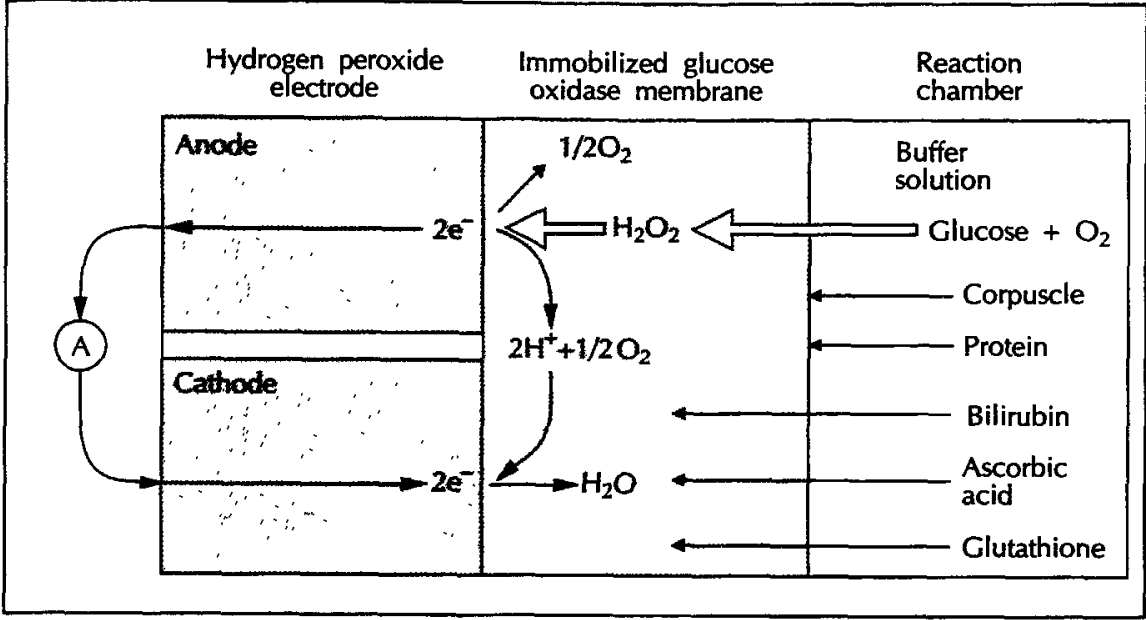

Fig. 3. A first generation enzymatic glucose sensor showing sensing and conversion elements. Hydrogen peroxide is generated from glucose and oxygen through the action of glucose oxidase immobilized to a membrane. This hydrogen peroxide reacts at the anode to produce two hydrogen ions, one oxygen atom and two electrons. The electrons are measured by an ammeter (A).

ing the operation of the FIA system, for example, by equipping it with on-line dialysis and segmented sample injection [11•]. A similar study used segmented sample injection, membrane-deposited reagent and membranes of low permeability. This system was used to determine I-lactate and creatinine in addition to glucose in whole blood samples [12]. Other studies have tried to eliminate interference [13] or to evaluate the amperometric determination of glucose in blood serum or whole blood [14].

As a result of these studies, application of biosensing FIA systems in the determination of glucose concentration for clinical purposes seems very attractive. The enzyme system can be immobilized, packed as a column and connected to the FIA system at a point before the sample passes through the detector, or immobilized directly onto the working surface of an electrode. In both cases, the reusability of the immobilized enzyme system effectively reduces operation costs by decreasing the consumption of enzyme. Other merits are the fast response time and the ability to alter the sample solution to meet the conditions optimal for the enzyme system so that longer operation periods are possible.

\section{Other clinical applications}

Analysis systems composed of a biosensor and an FIA system have many other applications in clinical analysis. The measurement of serum cholesterol, believed to be an important factor in coronary heart disease, has become a routine diagnostic analysis. An FIA system for determining serum cholesterol was designed using immobilized cholesterol esterase and cholesterol oxidase [15]. An anion exchange resin was used to remove materials causing interference prior to analysis in order to increase accuracy and precision. The accuracy of the results conformed to the guidelines of the national cholesterol education program of Canada.

An increase in the activity of serum creatine kinase is a valuable indicator for some health disorders including myocardial infarction, cerebral diseases, Duchenne's muscular dystrophy, Reye's syndrome and carbon mono- xide poisoning. This enzyme catalyzes a reaction that transfers the phosphate group of creatine phosphate to ADP. The ATP produced in this reaction can be used in the conversion of glucose into glucose-6-phosphate (G-6-P), catalyzed by hexokinase. The G-6-P is further converted to gluconolactone-6-P by G-6-P dehydrogenase and the coenzyme NADP. The reduced form of NADP (NADPH) produced in this reaction can then be determined spectrophotometrically or spectrofluorimetrically. In accordance with this reaction sequence, an immobilized enzyme column containing hexokinase and G6-P dehydrogenase, in conjunction with an FIA system, was used to determine the activity of creatine kinase in serum samples [16*]. The activity of two other enzymes that are valuable indicators of health, glutamate pyruvate aminotransferase [17] and lactate dehydrogenase [18], can also be determined using procedures that incorporate enzymatic reactions in series.

In most cases, the quantification of FIA operation is usually calculated by a univariate calibration based on the height or the area of the peak. For very complex biological samples with many interfering materials, however, a multivariate calibration system is more convenient. A photodiode array detector was applied to produce such a multivariate signal and this system was used to determine teniposide, an antitumor agent, in blood serum [19].

The components of urine can also be determined by a biosensing FIA system. Oxalate, a marker of calculosis, has been determined by a kinetic-enzymatic procedure [20]. The enzymes used in this analysis include oxalate decarboxylase and formate dehydrogenase; the former catalyzes the decarboxylation reaction of oxalate to produce formic acid, which is then converted to carboxylic acid by the latter. NADH is formed in this reaction and monitored spectrophotometrically at $340 \mathrm{~nm}$.

Although NADH itself is detectable by photometric or fluorimetric methods, the application of $\mathrm{NADH}$ oxidase to convert $\mathrm{NADH}$ into $\mathrm{NAD}^{+}$and produce hydrogen peroxide in the same reaction has been reported as a more sensitive way of determining NADH [21]. Higher sensitivity was obtained by mixing the hydrogen peroxide with luminol and potassium ferricyanide and measuring the emitted chemiluminescence with a photon counter. Us- 
ing this method, the detection limit of NADH is 10 pmol. It is possible to link this system to any NAD-dependent reaction and it has been used to determine magnesium in human serum by linking it to hexokinase and G-6-P dehydrogenase catalyzed reactions.

Techniques for determining several substrates simultaneously have also been investigated by using an arrangement of several immobilized enzyme columns in an FIA system. Glucose, ethanol and lactate in serum samples were measured simultaneously by a parallel configuration of three columns containing immobilized glucose

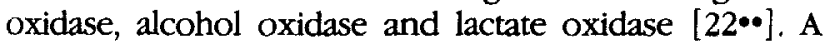
16-way switching valve was designed and adopted as the sample injection port for this system. An ascorbate oxidase column was used to pretreat the sample and a uricase column was connected before the immobilized enzyme columns to eliminate interference from ascorbic acid and uric acid. A schematic diagram of the multichannel FIA system is shown in Fig. 4.

Other clinically interesting analyses have been performed such as the determination of the concentration of bile acids in serum, which has been measured by a fluorimetric enzymatic FIA system [23] and a combination of FIA and liquid chromatographic processes [24]. Amino acids in serum have also been determined: L-alanine was measured using a packed-bed enzyme reactor comprising alanine dehydrogenase immobilized on poly(vinyl alcohol) beads [25]. Trace amounts of thiocyanate in saliva samples were measured by a kinetics spectrophotometric assay of a flow injection system [26].

\section{Determination of drug components}

Bio-field-effect transistors combined with an FIA system have been used to determine penicillin $G$, penicillin $V$ and cephalosporin $\mathrm{C}$ concentrations during a fermentation process [27] and gave results that matched well with off-line liquid chromatographic data. This was achieved by immobilizing penicillin $G$ amidase, penicillinase and cephalosporinase onto the gate of a pH-sensitive fieldeffect transistor. These bio-field-effect transistors for penicillin $\mathrm{G}$, penicillin $\mathrm{V}$ and cephalosporin $\mathrm{C}$ are usable for up to 3 months, 4 months and 1-2 days, respectively.

Other drugs including tetracycline, chlortetracycline, oxytetracycline, doxycycline and methacycline have been determined by FIA with a spectrophotometric detector [28] or a flow-through sensor [29]. The operation rate of the latter was emphasized as one hundred samples per hour. Berberine and benzethonium in drugs have been determined spectrophotometrically by batchwise and flow injection methods after a solvent extraction process [30].

\section{Applications in fermentation processes, the food industry and agricultural products}

Fermentation monitoring, as discussed further by Raju and Cooney (this issue, pp 40-44), is an essential part of bioprocess control. A schematic diagram for connecting fermenters with an FIA system is illustrated in Fig. 5. In this diagram, a recycling sampling system composed of a pump and a filter is shown. This design is suitable for a small jar-fermenter only. For a big fermenter for largescale production (e.g. $300 \mathrm{M}^{3}$ ton capacity) a sampling rate of a few milliliters every minute makes no difference to the final volume. Therefore, there is no reason to take the risk of contamination by recycling the sample solution. The dialyzer may be unnecessary if the flow system is designed to flush the surface of the sensor effectively or if the surface of the sensor is covered by materials that effectively obviate the adhesion of components of the medium.

Glucose is still the major compound of interest in the field of fermentation. A chemically modified graphite electrode with immobilized glucose dehydrogenase was used as the detector in an FIA system to determine glucose concentration in wine production [31 $]$. This system was reportedly used for 3 days of continuous operation with

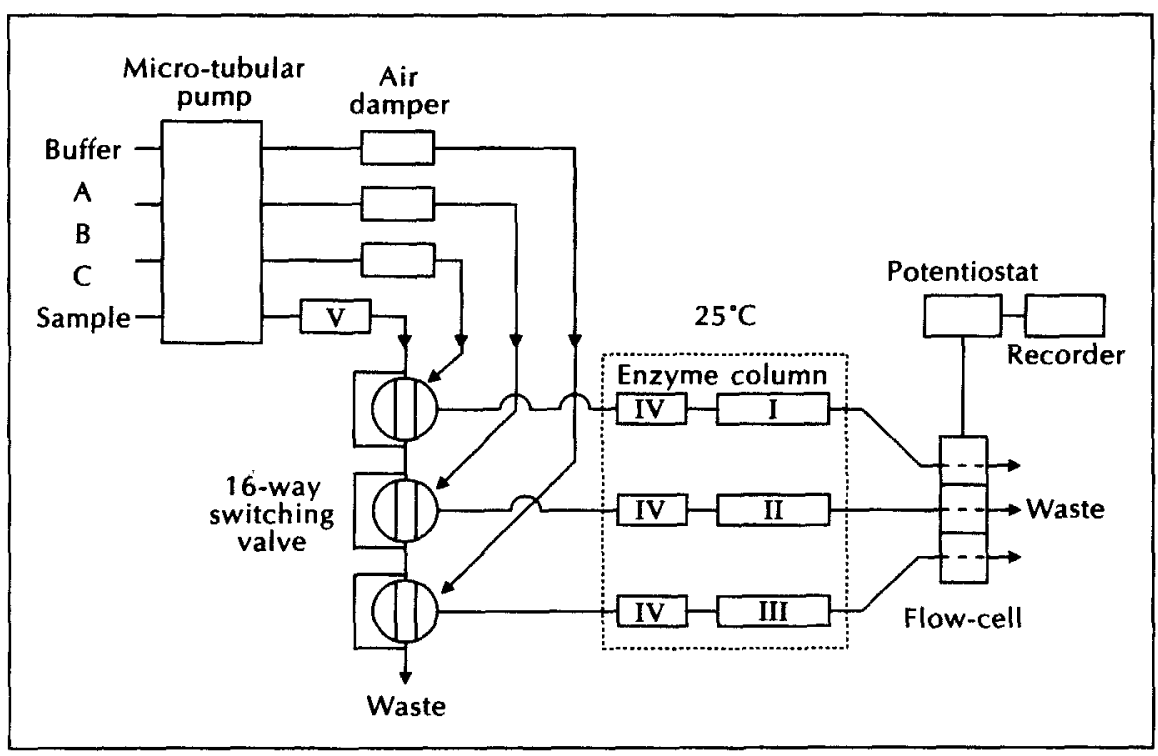

Fig. 4. Schematic diagram of a multichannel flow injection analysis system. I, lactate reactor; II, glucose reactor; III, ethanol reactor; IV, urate-elimInating reactors; $V$, ascorbate-eliminating reactor. Buffer $A$, phosphate $0.1 \mathrm{M}$, $\mathrm{pH} 8.0$; buffers $B$ and $C$, phosphate $(0.1 \mathrm{M}, \mathrm{pH} 7.0)$. 


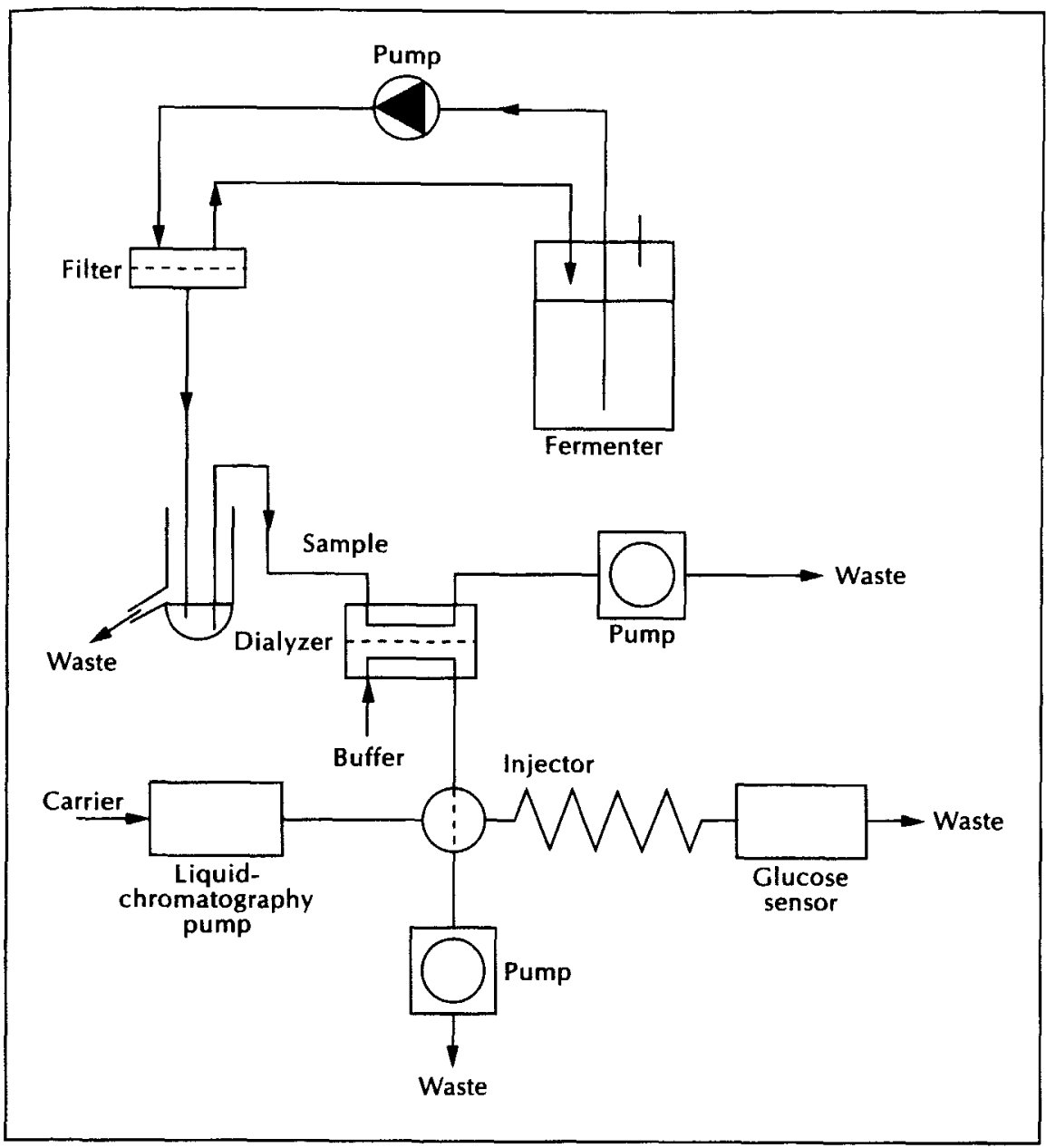

Fig. 5. Incorporation of a fermenter with a flow injection analysis system. no interference from alcohol observed. Another FIA system using a double injection process to measure glucose in a fermentation broth has also been reported [32]. The sample solution and enzyme solution were injected individually into a carrier stream. The hydrogen peroxide produced after passing a reaction coil was measured amperometrically.

Amino acids are another important class of compounds in fermentation. L-lysine is an important essential amino acid. Commercially, it is mainly produced by fermentation and used as an additive in fodder. The concentration of L-lysine in fermentation broths and fodder concentrate was determined with high specificity by a combination of an immobilized L-lysine monooxygenase column, a dissolved oxygen probe and an FIA system [33]. L-lysine was also measured using the same FIA and immobilized enzyme column, but with a different detection system: the hydrogen peroxide produced reacted with peroxidase, phenol and 4-amino antipyrine to produce a red quinoneimine dye which was detected spectrophotometrically at $500 \mathrm{~nm}$ [34] . L-glutamic acid, an important amino acid used to prepare monosodium L-glutamate, which is widely used in the food industry was measured, was by an amplification system based on substrate recy. cling [35*•]. L-glutamate was converted to 2-oxoglutarate by L-glutamate oxidase. The glutarate produced was subsequently converted back to L-glutamate by glutamate pyruvate aminotransferase. Using this method, sensitivity is increased 20-30-fold and concentrations of L-glutamate as low as 1 picomolar could be detected. L-alanine in serum and beverages can be determined by FIA with a fluorimetric detector [25]. Some other amino acids in cheese have been determined chemiluminescently by FIA [36].

Aspartame is an important artificial sweetener composed of aspartic acid and phenylalanine. The concentration of this dipeptide can be determined by FIA using two enzyme columns containing peptidase and aspartate aminotransferase, respectively, and an enzyme sensor with immobilized L-glutamate oxidase [37]. Aspartame is hydrolyzed into aspartic acid and phenylalanine by the peptidase. Aspartic acid is then converted into glutamic acid by aspartate aminotransferase and the product, L-glutamic acid, can then be determined by the glutamate sensor.

L-ascorbic acid is also an analyte that has attracted much attention. L-ascorbate oxidase converts ascorbate into dehydroascorbate. The hydrogen peroxide produced at the same time is detectable using a hydrogen-peroxide-sensing electrode. Ascorbate itself, however, is electroactive at the potential used to detect hydrogen peroxide. A system for ascorbate determination using FIA, an immobilized L-ascorbate oxidase column and a blank blank column with no enzyme was designed. On passing through the enzyme column some of the L-ascorbic acid was con- 
verted into the dehydro form and the decrease in response (measured against the blank column) was used to calculate the concentration of ascorbate [38]. Another system using cucumber juice as the carrier solution has also been used to determine ascorbate [39]. In still another system, measurement of the total ascorbic acid concentration has been reported using kinetic fluorimetric FIA and two serial injection valves [40].

Many carbohydrates have also been determined by FIA coupled to a biosensing system. In addition to fermentation broths (described above, [31•]), systems for measuring the concentration of glucose in milk [41] and alcoholic beverages $\left[22^{\bullet \bullet}\right.$ ] have also been described. In contrast with glucose oxidase, which oxidizes the $\mathrm{C}-1$ hydroxyl group of $\beta$-D-glucose, pyranose oxidase oxidizes the $\mathrm{C}-2$ hydroxyl group of the pyranose ring in hexoses and pentoses. This enzyme has been used in the determination of monosaccharides including glucose, xylose and galactose produced during the hydrolysis of cellulosic materials [42•]. This system can give a relative measurement of the total pyranose concentration in a sample of crude broth or an absolute value of an individual substrate in a sample without competing analytes. In another study, the determination of starch by FIA and a multi-enzyme system was reported [43•]. Amyloglucosidase, mutarotase and glucose oxidase were co-immobilized on a nylon membrane and bound to a platinum electrode. The concentration of starch in solution, when pretreated with $\alpha$-amylase at room temperature for one hour, was determined by measuring the hydrogen peroxide produced. In one recent report, an immobilized mannitol dehydrogenase column combined with FIA was used to detect D-mannitol (e.g. in celery and chewing gum) [44] and the $\mathrm{NADH}$ produced in this enzymatic reaction was measured fluorimetrically.

\section{Applications in environmental protection}

Environmental protection has become the focus of much attention in recent years. FIA systems equipped with biosensors have been used to measure indicators of environmental pollution, such as pesticides and herbicides. Paraoxon, an organophosphorus insecticide with a wide range of activity that is very toxic to mammals, was determined by both continuous flow and stopped flow systems in combination with an immobilized cholinesterase column. As an organophosphorus compound, paraoxon can form a stable complex with cholinesterase and inhibit its activity. Paraoxon determinations were made at a rate of 60 per hour [45.] on the basis of its ability to inhibit enzymatic hydrolysis of $\alpha$-naphthyl acetate and the subsequent reaction of the product, $\alpha$-naphthol, with p-nitro-benzenediazonium fluoroborate.

The concentration of phosphate in water is considered to be another important indicator of pollution. An amperometric FIA system with an immobilized enzyme reactor has been designed for determining phosphate in water samples [46-]. In the enzyme reactor, an amplification system for substrate recycling using purine nucleoside phosphorylase and alkaline phosphatase is co-immobilized along with xanthine oxidase. This amplification system increases sensitivity up to 12 -fold.

\section{Applications to microbiological and enzymatic reactions}

The activities of many enzymes have been determined using biosensing FIA systems, often by incorporating additional enzymes to convert the product formed by analyte catalysis into a detectable material. Two examples of this, as mentioned above, are glutamate pynuate transaminase (an indicative enzyme of liver function) [17] and lactate dehydrogenase. The activity of lactate dehydrogenase can be determined using an immobilized pyruvate oxidase membrane on an oxygen probe in an FIA system [47]. Another system that can determine the activities of lactate dehydrogenase and glutamate pyruvate transaminase simultaneously was developed by the same group and is based on measuring the pyruvate produced with pyruvate oxidase immobilized on an oxygen probe [48]. Guanase activity has been determined by measuring the hydrogen peroxide produced in the analyte-catalyzed reaction in conjunction with xanthineoxidase and uricase [49]. The FIA system used consisted of series-injection, stoppedflow and merging-zone methods. Another multi-enzyme FIA system, for determining the activity of creatine kinase in serum samples by photometric and fluorimetric detection has also been reported [50].

Microorganisms, bioreactions of microorganisms and cell components can also be monitored using biosensing FIA systems. The presence of Salmonella in food preparations causes severe food poisoning. To check for Salmonella in food products by the traditional method is time consuming, costly and often difficult because it is only present at very low concentrations. An FIA immunoassay system has been developed [ $51^{\bullet \bullet}$ ] for the purpose of detecting microorganisms belonging to this genus. A sandwich system composed of antibody, microorganism and glucose oxidase labeled antibody was constructed by immobilizing the antibody onto the inner wall of a Ty. gon tube through which the sample solution and enzyme labeled antibody solution were pumped in succession. Finally, a glucose solution was pumped through the tube and hydrogen peroxide determined electrochemically.

Another system, combining FIA with a membrane introduction tandem mass spectrometric system, was used to monitor liquid and gas components during the production of 2,3-butanediol by Bacillus polymyxa or Kleb siella oxytocain these microorganisms [52*•]. Although this study did not use a biosensor for measuring the analytes, we have included it because it was used to monitor a fermentation process.

An FIA system incorporating a fibre-optic biosensor to determine ATP and NADH has been designed [53*0]. Three enzymes, firefty luciferase, bacterial oxidoreductase and bacterial luciferase are co-immobilized onto a polyamide membrane. The first enzyme is used to measure ATP and the other two enzymes to measure NADH. 
By changing the reagents, ATP or NADH can be determined with the same sensing element. The activity of enzymes that produce ATP or NADH in their catalyzing reactions, for example kinase and dehydrogenase, can be determined by this system.

\section{Other applications}

A flow-injection biosensing system has been constructed using an immobilized alkaline phosphatase column, a planar $\mathrm{pH}$-sensitive field effect transistor and a flow in jection system. This system was used to determine the concentration of zinc(II) ions by activation of the immobilized cofactor-free apoenzyme in the column [54*•]. A chelating agent, 2,6-pyridine dicarboxylate, was injected between successive samples to regenerate the column.

Two immuno-FIA systems have been developed for determining methamphetamine [55*], a drug that is often abused, and trinitrotoluene (TNT) [56], an organic explosive. The latter involves mixing TNT with anti-TNT and determining the decrease of fluorescence of anti-TNT after mixing. The former incorporates immobilized albumin conjugated to methamphetamine on the surface of an AT-cut quartz crystal. The decrease of the resonant frequency of the crystal after contacting a solution of methamphetamine and monoclonal antibody against methamphetamine is measured, with the frequency decrease correlating with the concentration of anti-methamphetamine in the flowing solution. At a fixed concentration of anti-methamphetamine, the frequency decrease was found to be attenuated by the concentration of methamphetamine that was present in the solution. Concentrations of methamphetamine as low as $0.02 \mathrm{ppm}$ can be detected in about $5 \mathrm{~min}$.

\section{Conclusion}

The papers cited in this review demonstrate the wide ap. plicability of a combined biosensing FLA system to many fields including clinical diagnosis, the food industry, agricultural products and environmental control. The range and number of these systems is expected to increase as immobilization, flow injection and detection techniques improve. To analyze a biomaterial using traditional methods may be time consuming and even then the data may be of little value because the result is obtained hours or perhaps days after the sample was obtained. The acquisition of real-time, or almost-real-time, data is the most important advantage of biosensing FIA systems. Increasing use of this kind of system may be expected in the future.

\section{Acknowledgement}

The authors would like to express their appreciation to Dr Robert Wilson for helpful discussions and assistance with the English.

\section{References and recommended reading}

Papers of particular interest, published within the annual period of re new, have been highlighted as:

- of special interest

-. of outstanding interest

1. Clark LC, LYons C: Electrode Systems for Continuous Monitoring in Cardiovascular Surgery. Ann NY Acad Sci 1962, 102:29-45

2 FAN S, FANG Z: Compensation of Calibration Graph Curvature and Interference in Flow-injection Spectrophotometry Using Gradient Ratio Calibration. Anal Cbim Acts 1990 , 241:15-22

3 KOLEV SP, NAGY G, PUNGOR E: Mathematical Modeling of Single-layer Flow-injection Analysis System with Single-layer Enzyme Electrode Detection. Part 1. Development of the Mathematical Model. Anal Cbim Acta 1990, 241:43-53.

4. KOLEV SP, NAGY G, PUNGOR E: Mathematical Modeling of Single-layer Flow-injection Analysis System with Single-layer Enzyme Electrode Detection. Part $\Pi$. Stimulation of the Mathematical Model. Anal Chim Acta 1990, 241:55-69.

5. CHEN D, ZENG Y: Processing and Error Analysis of Signals in Flow-injection Analysis. Anal Chim Acta 1990, 235:337-342.

6. GUNASINGHAM H, TAN CH, Aw TC: Comparative Study of -. 1st-Generation, 2nd-Generation and 3rd-Generation Amperometric Glucose Enzyme Electrode in Continuous-flow Analysis of Undiluted Whole Blood. Anal Cbim Acta 1990, 234:321-330.

The three generations of electrodes are described, and the advantages and disadvantages of each in the analysis of biological samples are discussed. Second generation sensors seem best.

7. INMAN DJ, HORNLY WE:The Immobilization of Enzymes on Nylon Structures and Their use in Automated Analysis. Biochem I 1972,129.255-262

8 alves Da Silva M, Helena Gil M, Redinha JS, OUVEIRa Brett AM, COSTA PEREIRA JL: Immobilization of Glucose Oxidase on Nylon Membranes and its Application in a Flow-through Glucose Reactor. J Poly Sci [A] 1991, 29:275-279.

9 Trojanowicz M, Matsuzewsk W, Podsiadla M: Enzyme En. trapped Polypyrrole Modified Electrode for Flow-injection Determination of Glucose. Biosensors Bioelectronics 1990, 5:149-156.

Using electropolymerized polypyrrole films, enzymes can be immobilized onto the surface of electrodes of various shapes. A very thin membrane with a very fast response time can be created.

10. GEISE RJ, ADAMS JM, BARONE NJ, YACYNYCH AM: Electropolymerized Films to Prevent Interferences and Electrode Fouling in Biosensors. Biasensors Bioelectronics 1991, 6:151-160.

11 BUCH-RASMUSSEN T: Determination of D-Glucose in Undiluted

- Whole Blood Using Chemically Modified Electrodes and Segmented Sample Injection in a Flow System. Anal Chtm Acta 1990, 237:405-411

When analyzing whole blood samples, interference and fouling by the blood cells needs to be taken into consideration. This paper describes an FIA system with segmented sample injection and on-line dialysis to decrease dependence on the volume of the red blood cell fraction.

12. BuCH-Rasmussen T: Flow System for Direct Determination of Enzyme Substrate in Undiluted Whole Blood. Anal Chem $1990,62: 932-936$

13. MATSUZFWSKI W, TROJANOWICZ M, IEWENSTAM A: Elimination of Interference in Flow-Injection Amperometric Determination of Glucose in Blood Serum Using Immobilized Glucose Oxidase. Electroanalysis 1990, 2:607-610

14 Gunasingham H, TAN CH, Aw TC. Clinical Evaluation of Amperometric Enzyme Electrodes in Continuous-flow Analysis for Glucose in Undiluted Whole Blood. Clin Chem 1990, 36:1657-1661. 
15. CARPENTER A PURDY WC. The Determination of Total Serum Cholesterol by Flow-injection Analysis with Amperometric Detection. Anal Lett 1990, 23:425-435.

16. Fernandez-Romero JM, LuQue DE CASTRO MD: Determination of Creatine Kinase Activity Using a Co-immobilized Auxiljary Enzyme Reactor Coupled On-line with a Flow-injection System. Analyst 1991, 116:167-170.

The operation of multi-enzyme systems can be simplified by choosing enzymes that function in similar reaction conditions and co-1mmobilizing them in the same reactor. In this study an example is described for determining creatine kinase activity.

17. FERnaNDEZ-Romero JM, LUQUe DE CASTRO MD, VAlCARCEL $M$ : Determination of Alanine Aminotransferase in Human Serum in an Open-Closed Flow Injection Configuration. $J$ Biotech 1990, 14:43-52.

18. Tabata $M$, TOtanI $M$, MURachI $T$ : Determination of Lactate and Lactate Dehydrogenase Activity in Serum with the Flow Injection Analysis System Involving Immobilized Enzyme Column and Chemiluminescence. Anal Biochem 1991, 193:112-117.

19. Gerritsen MJP, Kateman G. Flow-injection System with Photodiode Array Detection and Multivariate Data Evaluation for Quantifying Teniposide in Blood Plasma. Anal Cbim Acta $1990,241: 23-50$

20. INFANTES JA, IUUQUE DE CASTRO MD, VALCARCEL M: Kinetic-enzymatic Determination of Oxalate in Urine by Flow-injection Analysis with Double-stopped Flow. Anal Cbim Acta 1991, 242:179-183

21. Murachi T, Totani $M$, IkEmodo $M$, Tabata $M:$ A Flow Injection Analysis System Involving Immobilized NADH Oxidase in Column Form for Clinical Analysis. J Biotech 1990, $14: 33-42$.

22. Matsumoto $K$, Matsubara $H$, hamada $M$, Ukeda $H$, Osajma $Y$ : - Simultaneous Determination of Glucose, Ethanol and Lactate in Alcoholic Beverages and Serum by Amperometric Flow Injection Analysis with Immobilized Enzyme Reactors. $J$ Biotech 1990, 14:115-126.

A parallel configuration of immobilized enzyme columns was used to determine several components in the same sample simultaneousty This procedure, though not a new one, is still a useful method for simultaneous determination of multiple analytes.

23. Membirla a lazaro F, luoue de Castro MD, Valcarcel M: Fluorimetric Enzymatic Flow-Injection Determination of Bile Acids in Human Serum. Fresenius J Anal Cbem 1990, 338:749-751.

24. Membiela a, lazaro F, Luque de Castro MD, Valcarcel M: Alternative Use of Flow-injection Analysis and the Combination of Liquid Chromatography and Flow-injection Analysis for the Determination of Total and Individual Bile Acid Concentration in Serum. Anal Chim Acta 1991, 249:461-467

25. KIBA N, TAGAMI H, FURUSAWA M: Determination of L-Alanine in a Flow-injection System with an Immobilized Enzyme Reactor. Anal Cbim Acta 1990, 239:307-310.

26. ANDERS BROE B, Elo HaRAID $H$ : Spectrophotometric Flow Injection Determination of Trace Amounts of Thiocyanate Based on Reaction with 2-(5-bromo-2-pyridylazo)-5-diethylaminophenol and Dichromate: Assay of the Thiocyanate Level in Saliva from Smokers and Non-smokers. Aralyst 1991, 116:647-651.

27. BRand U, REINHARDT B, RUTHER F, SCHEPER T, SCHUGERI K Bio-field-Effect Transistors as Detectors in Flow Injection Analysis. Anal Cbim Acta 1990, 238:201-210.

28. Alwarthan AA, Al-TamRaH SA, Sultan SM: Spectrophotometric Determination of Oxytetracycline by Flow Injection. $A n$ alyst 1991, 116:183-186.

29. LUU WZ, JIANG H, DU AZ: Determination of Tetracyclines by Flow Injection Analysis. Yaaxue Xuebao 1991, 26:391-394
30 SAKAI T: Solvent Extraction-Spectrophotometric Determination of Berberine and Benzethonium in Drugs with Tetrabromophenolphthalein Ethyl Ester by Batchwise and Flow Injection Methods. Analyst 1991, 116:187-190.

31. APPELQVIST R, HANSEN EH: Determination of Glucose in

- Fermentation Processes by Means of an On-line Coupled Flow-injection System Using Enzyme Sensors Based on Chemically Modified Electrodes. Anal Chim Acta 1990 235:265-271.

This paper outlines the standard model of a biosensing FIA system for monitoring fermentation.

32. WEN XW, Kipton H, POWELL J, ChRISTIAN GD, RuZICKA J Double Injection Enzymatic Flow Analysis of Glucose Using Amperometric Detection and an Oxygen-permeable Reaction Coil. Anal Cbim Acta 1991, 249:451-460.

33. Simonian AL, KHACHatrian GE, TATtKIAN SSH, AVAKIAN TSM, BADALAN IE: A Flow-through Enzyme Analyzer for Determination of L-Lysine Concentration. Biosensors Bioelectronics 1991, 6:93-99

34. POHLmanN a, STamm WW, Kusakabe H, Kula MR: Enzymatic Determination of L-Lysine by Flow-Injection Techniques. Anal Chim Acta 1990, 235:329-335.

35. YAO T, KOBAYASHI N, WASA T: Amperometric Flow-Injection - Anarysis of L-Glutamate Using an Immobilized Enzyme Reaction Amplification by Substrate Recycling. Electroanalysis $1990,2: 563-566$.

The response intensity of an analysis system could be amplified up to 30-fold by incorporate an enzyme that can convert the product of the reaction back to the substrate. This paper describes an example of this whereby glutarate is converted back to I-glutamate by glutamate pyruvate aminotransferase.

36. PuChades R, LEMIEUX L, SIMARD RE: Determination of Free Amino Acids in Cheese by Flow Injection Analysis with an Enzymic Reactor and Chemiluminescence Detector. I Food Sci 1991, 55:1555-1558.

37. Male KB, LuONG JHT, Mulchandan A: Determination of Aspartame in Dietary Food Products by a FLA Biosensor. Biosensors Bioelectronics 1991, 6:117-123.

38. GREENWAY GM, ONGOMO P: Determination of L-Ascorbic Acid in Fruit and Vegetable Juices by Flow Injection with Immobilized Ascorbate Oxidase. Analyst 1990, 115:1297-1299.

39. UChIYAMA S, SUZUKa S: Flow-injection Determination of Total Vitamin C Using Cucumber Juice Carrier. Bunseki Kagaku 1990, 39:793-797.

40. CHUNG HK, INGLE JD JR: Kinetic Fluorometric FIA Determination of Total Ascorbic Acid, Based on Use of Two Serial Injection Valves. Talanta 1991, 38:355-357.

41 PILIOTON R, MASCINI M: Flow Analysis of Lactose and Glucose in Milk with an Improved Electrochemical Biosensor. Food Chem 1990, 36:213-222.

42. Olsson L, Mandenius CF, VolC J: Determination of Mono- saccharides in Cellulosic Hydrolyzates Using Immobilized Pyranose Oxidase in a Continuous Amperometric Analyzer. Anal Chem 1990, 62:2688-2691.

Cellulosic hydrolyzates contain several kinds of fermentable sugars. Pyranose oxidase, the enzyme used in this case, can be used to determine a variety of monosaccharides in addition to glucose.

43. HAMID JA, MOODY GJ, THOMAS JDR: Multi-enzyme Electrodes

- for the Determination of Starch by Flow Injection. Analyst 1990, 115:1289-1295.

Enzymic biosensing systems are rarely used to determine complex molecules such as starch. This paper describes one such study in which pre-hydrolysis was used to obtain a measurable response from a multienzyme system.

44. KIBA N, INOUE Y, FuRUSAWA M: Flow-injection Determination of D-Manitol with Immobilized Mannitol Dehydrogenase. Anal Chim Acta 1991, 244:105-107. 
45. LEON-GONZALEZ ME, TOWNSHEND A: Flow-injection Determi- nation of Paraoxon by Inhibition of Immobilized Acetylcholinesterase. Anal Cbim Acta 1990, 236:267-272.

Toxic materials can be determined by virtue of their ability to inhibit the activity of biomaterials. In this case paraoxon was determined by its inhibition of cholinesterase catalyzed hydrolysis of $\alpha$-naphthyl acetate. This method has potential to measure toxic materials using living organisms.

46. YAO T, KOBAYASHI N, WASA T: Amperometric Flow-injection - $\quad$ System with an Immobilized Enzyme Factor for the Highly Selective Detection of Phosphate and On-line Amplification by Substrate Recycling. Anal Cbim Acta 1990, 238:339-343. A system for deterting phosphate in water samples and for increasing sensitivity to it by substrate amplification is described.

47. Okuma H, Sektmukai $S$, Hoshi M, Toyama K, Watanabe E: Biosensor System for Continuous Flow Determination of Enzyme Activities. 1. Determination of Glucose Oxidase and Lactic Dehydrogenase Activities. Enzyme Microb Tecb nol 1989, 11:824-829

48. OKuma H, Takahashi H, Sekimuka S, Watanabe E: Biosensor System for Continuous Flow Determination of Enzyme Activities. II. Simultaneous Determination of Plural Enzyme Activities. Enzyme Microb Technol 1991, 13:134-138.

49. TOMOda M, Uchida K, Higuchi N, SajI K, Satro S: Flow Injection Analysis of Guanase Activity. $J$ Flow Injection Anal 1990, 7:113-129.

50. LuQue DE CASTRO MD, FERNANDez-Romero JM: Photometric and Fluorimetric Determination of Creatine Kinase Activity by Using Co-immobilized Auxiliary Enzymes and an Open/Close Flow Injection Manifold. Anal Lett 1991, 24:749-765.

51. LUONG JHT, PRUSAK-SOCHACZEWSKI E: Development of a Flow -. Immunoassay System for the Detection of Salmonella ty phimurium. Anal Lett 1990, 23:1809-1826.

A sandwich type immunoassay system set up by immobilizing antibodies raised against $S$. typhimurium on the wall of a Tygon tube was used to detect microorganisms of this species. This immuno FlA system was reused more than 50 times.
52. HAYWARD MJ, KOTIAHO T, LISTER AK, COOKS RG, AUSTIN GD, -. NARAYN R, TSAO GT: On-line Monitoring of Bioreactions of Bacillus polymyxa and Klebsiella oxytoca by Membrane Introduction Tandem Mass Spectrometry with Flow Injection Analysis Sampling. Anal Chem 1990, 62:1798-1804. An outstanding method that can monitor major products and volatile metabolites during a fermentation process.

53. Gautier SM, Blum LJ, COULET PR: Multi-function Fibre-optic -. Sensor and the Bioluminescent Flow Determination of ATP or NADH. Anal Cbim Acta 1990, 235:243-253.

Two enzymatic systems for measuring different anabytes were co-immobilized on the same membrane to construct the sensing unit. By changing the reagents and reaction conditions, analytes can be se. lectively determined.

54. SATOH I, AOKI $Y$ - Biosensing of Zinc (II) Ions Using -. an Apoenzyme Reactor and an ISFET Detector in Flow Streams. Denki Kagaku 1990, 58:1114-1118.

A very clever idea for measuring the concentration of metallic ions is described in which determination relies on activation of an apoenzyme by binding the analyte as cofactor.

55. Miura N, Nigobashi H, Takeyasu A, Uda T, yamazoe N: Piezo- electric Crystal Immunosensor for Sensitive Detection of Methamphetamine. Chemical Sensor 1991, 7:53-56.

This study is a good example of applying immuno-FIA to the determ1nation of drugs of abuse.

56 Nakayama H, Sugihara H, Takahashi K, Miryazaki J, Mitsumata $T$ : The Development of a Sensor for Explosives Using an Immune Reaction. Chernical Sensor 1991, 7:57-60.

C-Y Chen, Research Center for Advanced Science and Technology, University of Tokyo, 4-6-1, Komaba, Meguro-Ku, Tokyo, Japan and Department of Agricultural Chemistry, National Taiwan University, 1, Sec. 4, Roosevelt Road, Taipei, Taiwan.

I Karube, Research Center for Advanced Science and Technology, University of Tokyo, 4-6-1, Komaba, Meguro-Ku, Tokyo, Japan. 